\title{
AUTOMATION OF FULL-SIZE WHEELCHAIR USER BODY 3D-SCAN DIMENSIONAL SIGNS REGISTRATION
}

\author{
K.K. Shcherbina ${ }^{1}$, E.V. Fogt ${ }^{1}$, M.A. Golovin ${ }^{1,2}$, M.V. Chernikova ${ }^{1}$, A.D. Kuzicheva ${ }^{1}$ \\ ${ }^{1}$ Albrecht Federal Scientific Centre of Rehabilitation of the Disabled (Federal State Budgetary Institution "Federal Scientific Center \\ of Rehabilitation of the Disabled n. a. G.A. Albrecht" of the ministry of Labor and Social Protection of Russian Federation), \\ St. Petersburg, Russia - fogtlisbet11@yandex.ru, muxagolovin@gmail.com \\ ${ }^{2}$ Peter the Great St.Petersburg Polytechnic University (SPbPU), St.Petersburg, Russia
}

KEY WORDS: Rehabilitation, Clothes, 3D-scan, Algorithm, Size, Disabled, Distance technology, Automatization.

\begin{abstract}
:
Distance clothing technology is an actively developing area. For its implementation in the highly specialized area of manufacturing technical means of rehabilitation, and, in particular, in the manufacture of special functional and aesthetic clothing for disabled people, it is necessary to solve organizational and technical issues. An example of a technical issue is remote acquisition of dimensional features. The dimensional characteristics of the human body are an integral part of the technological process of manufacturing individual clothing. The use of 3D scanning makes it possible to implement remote technology for individual design and manufacture of clothing. The production of clothing for wheelchair users involves the adaptation of standard clothing design techniques to the specific properties of the posture. A case of a patient with a C5-C6 cervical vertebra fracture who has been using a wheelchair for more than 25 years is considered. The study used 3D human models obtained with a 3D scanner. The technique of scanning and an example of processing the obtained data are presented. The main features of dimensional features have been determined and an algorithm for their determination by anatomical landmarks has been developed. Recommendations are given for processing 3D scans and combining them into one 3D model. It is shown that the use of 3D scanning for the remote production of a set of functional and aesthetic clothing for a wheelchair user is a way to produce comfortable individual clothing.
\end{abstract}

\section{INRODUCTION}

Analysis of various Internet technologies for the manufacture and purchase of clothing showed that in order to improve the quality of manufacturing special clothing for the disabled and the effectiveness of creating new models of it, it is possible to improve the existing remote technology for manufacturing such clothing as follows:

- to increase the completeness of the initial information about the patient for designing clothes, provide the ability to obtain an electronic geometric model (scanator) of his figure by 3D scanning. In this case, it is necessary to first apply the markings on the body surface using markers and rubber bands with special markings;

- to ensure the interaction of the scanator and the process of constructing a patient dummy based on it, including an asymmetric dummy;

- to improve the accuracy of the calculation and construction of drawings of the basic design of special clothing and its transformation into a model design, provide the possibility of its design by two methods: firstly, 2D CAD, and secondly, 3D CAD with the subsequent deployment of patterns on a plane without using indirect 2D design methods;

- to increase the efficiency of optimization of the basic and model designs of special clothing, to provide an opportunity in the process of its manufacture to try on the product on a physical mannequin made or adapted from a 3D scan of the consumer's figure, while observing strict confidentiality of patient data;

- to provide the possibility of designing a 3D model of clothing based on this mannequin and carry out its virtual fitting on a 3D patient's mannequin.

Comparison of the technical characteristics of individual modules and 3D scanning devices allows us to draw the following conclusions:

- it is possible to ensure high accuracy of scanning a person (up to $0.5 \mathrm{~mm}$ );
- it is possible to provide instant scanning of a person, in which human movements (involuntary) will not introduce an error in the scan result;

- it is possible to record textures, that is, to register marks, overlays and other objects on a person;

- it is possible to combine various shooting technologies in one scanning device;

- it is possible to obtain the required dimensions from the scanner in an automated mode.

However, there are the following restrictions:

- at present it is impossible to provide simultaneously instant scanning and high scanning accuracy;

- automatically received dimensional characteristics differ from those registered manually, which requires a change in clothing design methods.

Obtained sizes of the human body are the essential part of the technological process the individual clothing producing, notably, functional special clothing for the disabled. There are different means and methods to record them. But its use leads to variety of actual values.

The main problem is that the data, even if it was registered with automated method, is needed to be checked by specialist. Development of a digital tool for analysis is aimed to improve distance clothing technology (Smirnova et al., 2020).

Many modern CAD systems for sewing products combine threedimensional and two-dimensional technologies. "Unfolding" clothing CAD (3D $\rightarrow 2 \mathrm{D})$ allows to design the shape of the product in three-dimensional space, and then get the product details on the plane for further transformation. In such systems, the initial information is set in the form of a mathematical model $(\mathrm{MM})$ on the surface of a typical mannequin or figure, which is converted into MM of the product surface and then proceeds to two-dimensional scans, from which the parts of the product are formed. "Dressing" clothing CAD $(2 \mathrm{D} \rightarrow 3 \mathrm{D})$ are developed for designing flat product patterns using traditional planar methods and further "stitching" and "dressing" them on a virtual three- 
dimensional mannequin to check the fit of the product and make changes to the flat patterns. In addition, there are mixed systems $2 \mathrm{D} \rightarrow 3 \mathrm{D} \rightarrow 2 \mathrm{D}$, where both technologies are used with some limitations.

There are a variety of three-dimensional clothing CAD that allows to design and visualize clothing models according to the figure. At the same time, the figure can be built both according to the dimensional characteristics of the client, and according to an electronic geometric model obtained by 3D scanning. The main difficulty lies in the fact that the classic mannequin, to work with which the functionality of the programs is directed, laboriously adapts to the figure of the patient, who is indicated with functional clothing.

The aim of the study was to develop an algorithm for obtaining dimensional characteristics of a full-size human model obtained by $3 \mathrm{D}$ scanning a patient for the design of special clothing.

As example of how to measure human 3D-scan there is an online software 3D Measure Up (ProtoTechSolutions, India). It allows to form measurements of a full-size human electronic geometric model (EGM). Figure 1 shows the working window of the program with the result of measuring the human EGM, which is obtained by automatically constructing dimensional features.

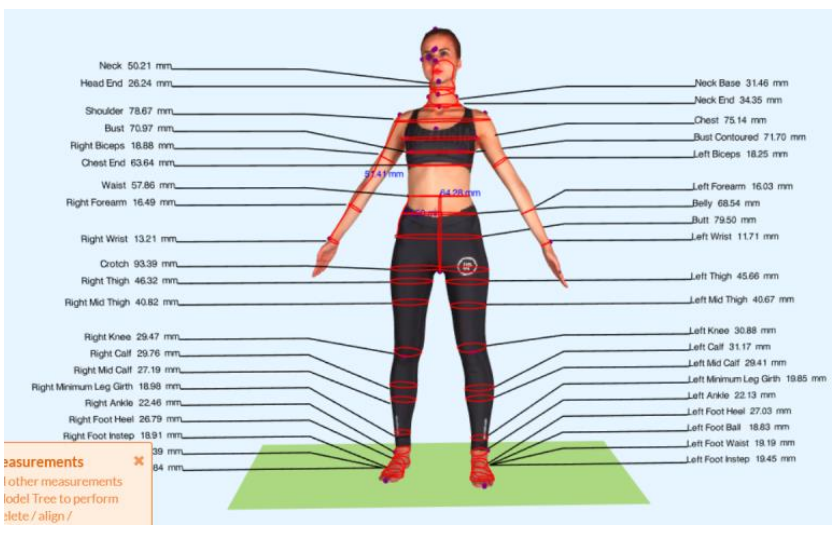

Figure 1. Program Window 3D Measure Up (ProtoTechSolutions, India)

The system can automatic orientate the 3D model, but there are some limitations of the 3D model analyzing:

1) automatic analysis only for vertical pose;

2) measurement of angular dimensions is not provided;

3 ) it is not possible to measure a linear size projection, like the distance between the frontal planes passing each through a reference point by constructing a section in the sagittal plane of the body EGM and measuring the perpendicular to the frontal plane as the distance between frontal planes;

4) the need for each loaded EGM to re-build the necessary dimensions that are not available in the program.

\section{METHODS AND MATERIALS}

The study used 3D human models, captured with 3D scannerStructure sensor, Occipital, Canada. The anonymous experimental data for the study were obtained by the Federal Scientific Center of Rehabilitation of the Disabled named after G.A. Albrecht. The study was performed in accordance with the ethical standards presented in the Declaration of Helsinki. The study protocol was reviewed and approved by the local expert collegiate council before the beginning of the study. The study involved a patient with a fracture of the cervical vertebrae C5C6, using a wheelchair for more than 25 years. Patient provided his written informed consent prior to his inclusion in the study. The 3D-scans were merged using Meshmixer (Autodesk Inc.) and MeshLab (Consiglio Nazionale delle Ricerche). The following software products were used to analyse 3D-scans: Rhinoceros 6 and the Grasshopper plugin (McNeel). Methods of geometric modeling and linear algebra were used.

\section{STUDY}

Use of 3D-scanning allows to implement the distance technology of individual clothing design and producing. This technology allows to solve a number of problems of the organization of remote data collection about the customer and his anatomical and physiological features. For example, the objectivity of manual registration of dimensional characteristics (measurement) of a disabled person is questioned due to the various skills of specialists who directly perform measurement, and the use of photographing distorts the perception of the figure.

Advantages of 3D scanning for the purpose of implementing distance clothing technology:

1.the objectivity of registering the coordinates of surface points, 2.the ability to record the color of the scanned object

(registering textures),

3.for some technologies - almost instantaneous shooting.

Structuring the work of a specialist by drawing up a sequence of operations allows him to organize his work activity with a certain degree of compliance with the actual process of interaction with the customer. The individuality of each consumer in the provision of functional aesthetic clothing allows us to offer a basic version of the algorithm for the work of a specialist.

The main criteria by which the sequence of obtaining information is built is the ability of the consumer to keep a fixed position in the process of collecting information and the degree of technological support of a specialist. If the patient cannot maintain a natural posture during filming, then even the presence of an accurate and fast 3D scanner will not allow using it.

At the same time, there are certain limitations. Manufacturing the clothes for wheelchair users assumes the adaptation of standard methods projecting clothing for pose peculiar properties. So the location of point $\mathrm{M}$ (patella center) varies in standing and sitting positions. Its location has influence on registration of such dimensions as knee girth, trousers' length on the side in sitting position. Setting of point $M$ to the model is the starting point for building the dimensions mentioned above. Knee girth is registered also on posterior side of knee, trousers' side length in the sitting position - on lateral hip side. Accordingly, for constructing the individual dimensions it is necessary to create a line on surface and then define its length. In this connection, given the need to register a person in a sitting position for correct registration of the point $\mathrm{M}, 3 \mathrm{D}$-scanning of the areas of interest is difficult. This is due to the landing of the user in the wheelchair: posterior surface of the leg (the calf) is located close to the seat of the wheelchair, and the armrest overlaps the hip. At the same time, if the design of the wheelchair and the equipment used allows, it is theoretically possible to scan the back of the knee. To shoot the hips, you need to move or remove the armrest. In this case, the solution of the problem may lead to the lack of support for user on the armrest, which will cause tension of the torso. Accordingly, dimensions based on the upper body and torso will not reflect the actual location of the wheelchair user.

Measurement and scanning for the purpose of subsequent measurement of the digital materials obtained is possible only if the subject has all the technical means of rehabilitation provided for in the individual program of rehabilitation and abilitation (IPRA) (including colostomy bags, etc.).

The basic requirements for a disabled person's clothing are as follows. During shooting the person being scanned must wear a tight, oversized T-shirt and thin knitted leggings or shorts. 
Clothing should not create excessive volume, which will reduce the accuracy of measurements. Clothing should not be tight, unless otherwise stated in the IPRA.

It is necessary to have the following special devices for marking the measurement points when scanning on the measured object: - an elastic band to indicate the waist line, located along the natural waist line, since it is acceptable and more consistent with the requirements of the construction of the structure than indicated in the national standards;

- a device of the "staple" type for scanning difficult places (armpits and groin);

- on the scanned item, if it is necessary to have pockets and / or structural fixation elements in its functional aesthetic clothing, their position must be fixed and recognizable on its threedimensional model;

Additionally, the following initial data should be marked and recognized on a three-dimensional model:

- product length (shoulder and waist separately);

- desired sleeve length.

In this regard, the scanning should be performed in stages. So, it is needed to analyse the diagnosis of the customer, the number of residual functions, the features of the figure and physique, certainly taking into account the dimensions that will be registered in the future on the $3 \mathrm{D}$ model.

On the basis of this information, the following scans can be taken: 1.sequentially individual body segments;

2. sequentially the upper torso and lower torso;

3.with alternating scanning sections, which are captured during isotonic muscle contraction of other body segments.

At the first step it is needed to crosslink separate scans (Figure 2). The head of the patient is not showed. Legs are on footrests. From left to right:

1.normal sitting position;

2.abnormal sitting position without armrests, arms on the hips, wheelchair user is bearing against the seatback;

3.abnormal sitting position without right armrest, right arm on the right hip;

4.abnormal sitting position with a metal ruler in the right armpit, the body is tilted to the left;

5.abnormal sitting position, the body is tilted forward, wheelchair user is bearing against the armrests.

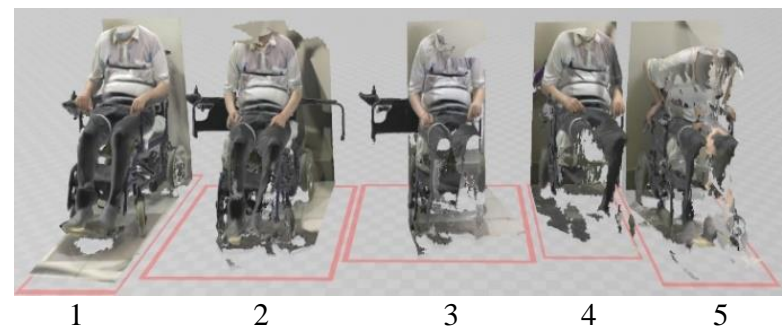

Figure 2. 3D scans (patient in different positions)

Subsequent cleaning of scans, their correct spatial location and crosslinking allows you to get a model ready for the placement of anatomical landmarks and registration of dimensional features (Tan Xiaohui et al.,2018).

All 3D-scans were oriented and matched by the wheelchair details. After that, foreign objects were removed from 3D scanning area (wheelchair, ruler, floor and wall). The movements of the wheelchair user during 3D-scanning and between 3Dscanning were also taken into account and processed. The scan of legs was restored from the scans 1-3, arms - from scan 2, back - from scan 5. The scan 4 was used to place anatomical landmarks. After that the 3D-model is needed to be analysed for defects (Figure 3). The inner surfaces of the legs should be located separately from each other, not connected, the back surfaces of the knees, hips, waist and back should be closed, the position of the upper limbs should ensure the removal of the necessary girths and not merge in these areas with the body or legs.

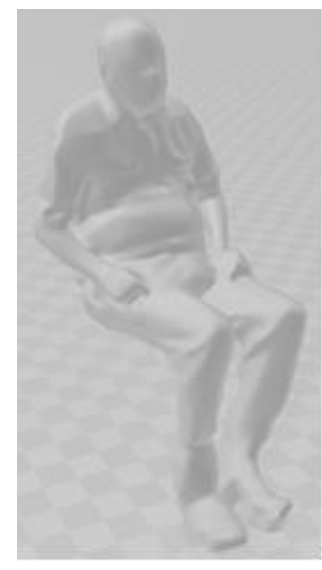

Figure 3. 3D model after processing

The work in the software module for the analysis of an electronic geometric model consists of the following stages:

1) preliminary stage - preparation of the model;

2) model scaling stage;

3) model orientation stage;

4) the stage of the location of anatomical landmarks for taking dimensions;

6) the stage of analyzing the sizes;

7) the stage of downloading the measurement values.

The basic principles that form the basis of the developed algorithm for EGM analysis:

1) the ability to quickly navigate in three-dimensional space;

2) preliminary location of anatomical landmarks;

3) fast measurement formation.

The algorithm of the EGM analysis software module, written in pseudocode, is presented in table 1 .

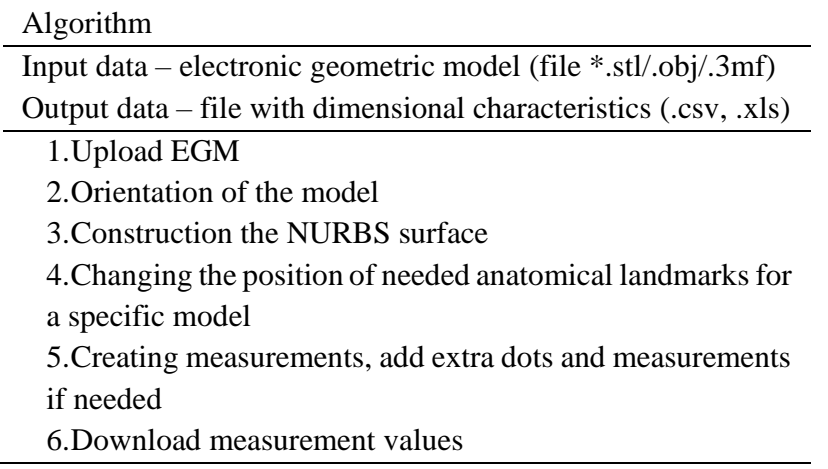

Table 1. Algorithm of program module

To develop an algorithm characteristics of the sizes used in the design of clothing were structured.

Type 1. Height, distance, anteroposterior diameter, width, etc. Represents:

1.measuring the distance from any point of the model to the surface (vertical, horizontal), for example, the height from the waist to the floor;

2.measurement of the projection length (on the coordinate axis) between two points. 
Type 2. Girth (waist, neck, etc.) Two-point section on the model surface

Type 3. Measurement along the section. Summed measurement of parts of cross-sections at several points

Type 4. Length (shoulder, etc.) Measurement of length along the surface of the model between two points

Type 5. Combined size. Measuring the length of the model surface by 3 or more points.

Type 6. Angle. Angular dimension between the horizontal plane and the section plane

At the figure 4 it is shown a small part of an algorithm to measure combined sizes in visual interpretation.

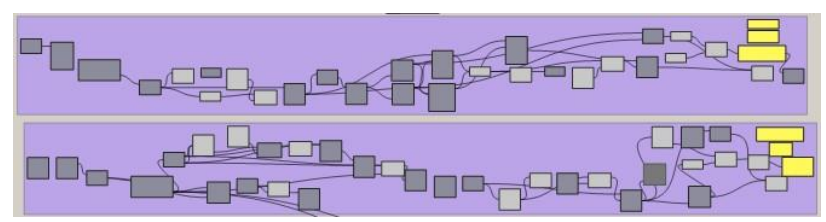

Figure 4. Part of an algorithm in Grasshopper

The template in Rhinoceros includes all anatomical landmarks for standard body measurements: circumferences, lengths, diameters, etc. Besides specially to analyze sitting pose there are some extra dots to place on EGM. Dots are used to plot a line, curve or to orient a plane. Examples are: line - anterior-posterior thoracic diameter, curve - chest breadth. Planes are needed to cut a model and measure a part of it and to plot special dots, lines and curves. Also, it is possible to project cross sections of the scan to the plane and use it to plot a complex size. It is needed for sizes such Back neck point to waist (5.4.5 according to ISO 85591:2017).

Automatic construction of dimensional characteristics based on the resulting $3 \mathrm{D}$ model is performed by using mathematical operators and functions. Curves on the surface of 3D-scan can be built such as minimal distance between anatomical landmarks but it will not match with ISO requirements so it is not right sometimes. To solve this, different mathematical functions are used simultaneously to plot different variants of a size and the logic equations to choose right size.

It takes about 15 seconds to evaluate the 3D-scan to plot all dimensional characteristics. So, in practice, the number of needed sizes is much less (about 30 against all possible) and the evaluation time is 5 seconds.

In the developed module, it is possible to register male and female sizes for both the right and left sides of the human body. EGM with sizes is shown at figure 5.

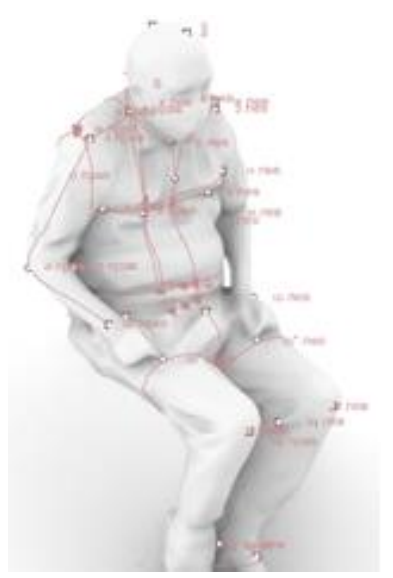

Figure 5. 3D model with marked anatomical landmarks and sizes
Red lines show the different sizes on a 3D-model. In particular, thigh girths are shown, outer arm length, back neck point to wrist length, total crotch length and other. Each anatomical landmark is shown with unique id. But the sizes do not have id because combined sizes use a number of curves and/or lines. That is why it is not correct to overload the workspace of an algorithm. Example of registered sizes is shown at figure 6.

$$
\begin{aligned}
& \text { Bзу_T11_лев } 837.55 \\
& \text { Oш_T13 } 18.65 \\
& \text { Or1_T14 } 1172.45 \\
& \text { Or2_T15 } 1160.45 \\
& \text { Or3_T16 } 1152.35 \\
& \text { Oт_T18 } 1159.1 \\
& \text { Влт_T7 } 696.8
\end{aligned}
$$

Figure 6. Example of registered sizes

This makes it possible to take more into account of the features of the customer's figure and further visually and aesthetically compensate them in the design of clothing, without compromising the functions inherent in it.

Also, if the customer's mobility allows, it is possible to scan in a wheelchair, in which scanning of the figure and body segments interest areas is available. Such device can be equipped with parts that are transparent for the used scanning device, as well as spread the structural elements of the wheelchair to the sides so that they do not limit the working area of the scanner.

The advantages of the proposed method include:

1.the possibility of automated measurement of the customer even in sitting position;

2.the ability to subsequently manufacture other products to the customer without his presence - by recording the dimensions according to the already processed scan;

3.the ability to analyze the body shape in case of repeated customer calls;

4.the possibility to add unique measurements, save them and easy use to the next customer.

\section{CONCLUSION}

The case of using 3D scanning for distance manufacturing of a set of functional and aesthetic clothing for a wheelchair user was considered. The technology of laser manual 3D scanning with texture recording is applicable for highly specialized tasks of clothing manufacturing.

At the same time, the scanning process itself is fully creative and should take into account not only the features of the equipment, but also the medical characteristics of the customer, and the features of the technical means of rehabilitation that he uses. The model linked from separate parts can be correctly analysed for the purpose of obtaining dimensional features and subsequent production of special functional and aesthetic clothing for the disabled.

Understanding the purpose of processing the model allows to correctly connect individual scans and get objective individual dimensions.

The study will continue in the directions of the anatomical landmarks automated construction and reduction of 3D-scanning time.

\section{ACKNOWLEDGEMENTS}

The authors are grateful to colleagues at the Albrecht Centre for their support in this study. 
The study was carried within state task № 149-00006-20-02 from 07.02.2020

\section{REFERENCES}

ISO 8559-1:2017 Size designation of clothes. Part 1: Anthropometric definitions for body measurement

Rhinoceros. https://www.rhino3d.com/ (3 September 2020).

Smirnova L.M., Volkova V.M., Golovin M.A., Karabanova $\mathrm{N}$.Yu. Technologies for manufacturing and purchasing clothing for the disabled: solutions for improving the author's remote technology. Journal of Physical and Rehabilitation Medicine, 2020, Vol. 2, No. 4, 20-33

Tan Xiaohui, Peng Xiaoyu, Liu Liwen, Xia Qing. Automatic human body feature extraction and personal size measurement Journal of Visual Languages \& Computing. Vol. 47. 2018, Pages 9-18. https://doi.org/10.1016/j.jvlc.2018.05.002.

Webtool 3D Measure Up, ProtoTech Solutions https://3dmeasureup.com/ (15 April 2020) 